\title{
Intravenous infusion of mesenchymal stem cells for protection against brainstem infarction in a persistent basilar artery occlusion model in the adult rat
}

\author{
Ai Namioka, MD, PhD, ${ }^{1}$ Takahiro Namioka, MD, PhD, ${ }^{1}$ Masanori Sasaki, MD, PhD, ${ }^{1-3}$ \\ Yuko Kataoka-Sasaki, MD, PhD, ${ }^{1}$ Shinichi Oka, MD, PhD, ${ }^{1}$ Masahito Nakazaki, MD, PhD, ${ }^{1}$ \\ Rie Onodera, PhD, ${ }^{1}$ Junpei Suzuki, PhD, ${ }^{1}$ Yuichi Sasaki, PT, PhD, ${ }^{1}$ Hiroshi Nagahama, PhD, ${ }^{1}$ \\ Jeffery D. Kocsis, $\mathrm{PhD},{ }^{2,3}$ and Osamu Honmou, MD, PhD ${ }^{1-3}$
}

\begin{abstract}
1Department of Neural Regenerative Medicine, Research Institute for Frontier Medicine, Sapporo Medical University School of Medicine, Sapporo, Japan; ${ }^{2}$ Department of Neurology, Yale University School of Medicine, New Haven; and ${ }^{3}$ Center for Neuroscience and Regeneration Research, VA Connecticut Healthcare System, West Haven, Connecticut
\end{abstract}

\begin{abstract}
OBJECTIVE Morbidity and mortality in patients with posterior circulation stroke remains an issue despite advances in acute stroke therapies. The intravenous infusion of mesenchymal stem cells (MSCs) elicits therapeutic efficacy in experimental supratentorial stroke models. However, since there are few reliable animal models of ischemia in the posterior circulation, the therapeutic approach with intravenous MSC infusion has not been tested. The objective of this study was to test the hypothesis that intravenously infused MSCs provide functional recovery in a newly developed model of brainstem infarction in rats.
\end{abstract}

METHODS Basilar artery (BA) occlusion (BAO) was established in rats by selectively ligating 4 points of the proximal BA with 10-0 nylon monofilament suture. The intravenous infusion of MSCs was performed 1 day after BAO induction. $\mathrm{MRI}$ and histological examinations were performed to assess ischemic lesion volume, while multiple behavioral tests were performed to evaluate functional recovery.

RESULTS The MSC-treated group exhibited a greater reduction in ischemic lesion volume, while behavioral testing indicated that the MSC-infused group had greater improvement than the vehicle group 28 days after the MSC infusion. Accumulated infused MSCs were observed in the ischemic brainstem lesion.

CONCLUSIONS Infused MSCs may provide neuroprotection to facilitate functional outcomes and reduce ischemic lesion volume as evaluated in a newly developed rat model of persistent BAO.

https://thejns.org/doi/abs/10.3171/2018.4.JNS173121

KEYWORDS transplantation; mesenchymal stem cell; stroke; vascular disorders

$\mathrm{T}$ The human brain has a higher proportion of white matter relative to gray matter, ${ }^{7}$ indicating the potential importance of white matter preservation after stroke. The majority of preclinical drug therapies examined in rodent models have targeted gray matter. ${ }^{35}$ However, the white matter/gray matter ratio in rodents is lower than that in humans, ${ }^{7}$ which is a possible reason for the unfavorable translational results of many clinical studies of drug candidates for stroke that were favorable in rodent studies. ${ }^{7,27}$ Nonhuman primate models have been used to test the effects on cerebral ischemia, including white matter injury, to address these barriers in the translation of results from animal research to humans. However, the cost and limited availability of nonhuman primates might not allow for sufficiently powered definitive efficacy studies. 7,27

The brainstems in humans and rodents have abundant white matter tracts, ${ }^{13,36}$ and white matter has lower infarction thresholds for ischemic injury than gray matter. ${ }^{4}$ Outcomes in patients with posterior circulation stroke remain poor, even after advanced acute stroke therapies. ${ }^{31}$ Thus, brainstem infarction could be a potential experimental

ABBREVIATIONS ACB = anterior cerebellar branch; $\mathrm{AICA}=$ anterior inferior cerebellar artery; $\mathrm{BA}=$ basilar artery; $\mathrm{BAO}=$ basilar artery occlusion; FITC = fluorescein isothiocyanate; $\mathrm{MCAO}=$ middle cerebral artery occlusion; $\mathrm{mNSS}=$ modified neurological severity score; $\mathrm{MSC}=$ mesenchymal stem cell.

SUBMITTED December 13, 2017. ACCEPTED April 5, 2018.

INCLUDE WHEN CITING Published online October 12, 2018; DOI: 10.3171/2018.4.JNS173121. 
target to test the therapeutic efficacy of drug candidates. There are few available reliable animal models of ischemia in the posterior circulation. The middle cerebral artery occlusion (MCAO) model in rats is the most widely representative animal model of stroke. ${ }^{8,26}$ However, several models of endovascular suture, clipping, permanent sutured ligation, and photochemical or electrocautery occlusion of vertebral and basilar vessels in animals have been reported for several species, including rats, gerbils, cats, and dogs. ${ }^{13,14}$

The intravenous administration of mesenchymal stem cells (MSCs) after supratentorial stroke in MCAO models provides therapeutic efficacy for reducing lesion volume and providing functional improvement. ${ }^{9-11,21,22,29,34}$ Suggested therapeutic mechanisms of MSCs include secretion of neurotrophic factors that provide neuroprotection, neurogenesis, neovascularization, and synaptogenesis and induce axonal sprouting. ${ }^{10,28,29,32}$

Here, we established a persistent brainstem infarction model in rats confirmed using MRI by selectively occluding 4 points of the proximal region of the basilar artery (BA), and we investigated the therapeutic efficacy of the intravenous infusion of MSCs in this BA occlusion (BAO) model system. Evaluations of the behavioral outcomes, MRI findings, and histological analyses were performed after the MSC infusion.

\section{Methods}

The animal care and use committee of Sapporo Medical University approved the use of the animals in this study; all procedures were performed in accordance with institutional guidelines.

\section{BAO Model}

Female Sprague-Dawley rats $(n=42$; weight $250-300$ g) were used in the BAO model. The animals were anesthetized with an intraperitoneal injection of ketamine (75 $\mathrm{mg} / \mathrm{kg}$ ) and xylazine (10 $\mathrm{mg} / \mathrm{kg})$. They were placed supine, and a $2-\mathrm{cm}$ midline neck incision was made through the subcutaneous tissue and platysma (Fig. 1A). The edge of the left sternohyoid muscle was retracted medially (Fig. $1 \mathrm{~B})$ and the rostral-most part of the longitudinal muscles was detached from the skull base by midline division (Fig. 1C). The ventral surfaces of the foramen magnum, clivus (basilar portion of the occipital bone), and occipital condyle were then exposed (Fig. 1D). A minimal anterior clivectomy was started from the anterior rim of the foramen magnum using a fine rongeur. A small bone window was created through the clivus (Fig. 1D and E). The dura mater was opened using a straight incision to expose the ventral aspect of the brainstem (Figs. 1F and 2A).

The BA was occluded at 4 points with a 7-mm sterile 10-0 nylon monofilament suture (Bear Nylon Suture, Bear Medic Corp.). After occluding the 4 points of the BA just caudal and rostral to the anterior inferior cerebellar artery (AICA) and anterior cerebellar branches (ACBs) that symmetrically branched out from the trunk of the BA (Figs. $1 \mathrm{~F}, 1 \mathrm{G}$, and 2D), we confirmed the absence of anterograde flow to the occluded BA with a tail vein injection of fluorescein isothiocyanate (FITC)-dextran $\left(2 \times 10^{4}\right.$ molecular weight, $0.5 \mathrm{ml}$ of $0.6 \mathrm{mg} / \mathrm{ml}$, Sigma-Aldrich) using a fluorescence microscope (Axio Zoom V16, Carl Zeiss; Fig. 2). Fluorescent images in the arterial phase showed no-flow areas due to the occluded BA, indicating the infarcted lesion (Fig. 2E and F). At the bottom of the occlusion site,
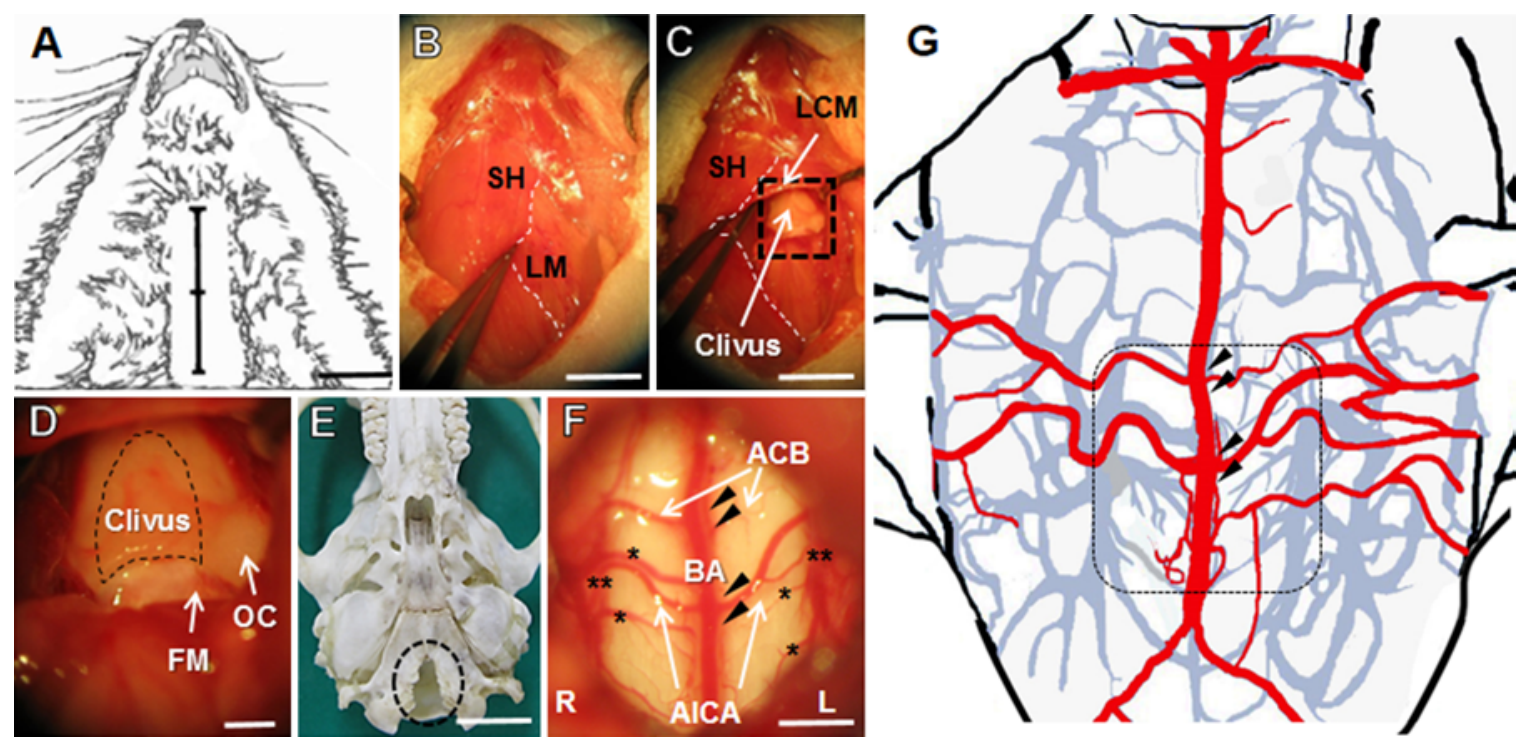

FIG. 1. Operative procedure of BAO in a rat. A: A 2-cm skin incision is indicated by a marker. B: The edge (dashed line) of the left sternohyoid muscle (SH) is retracted medially with fine forceps to expose the longitudinal muscles (LM). C: The longus capitis muscles (LCM) were divided to expose the clivus. D: Boxed area in panel C. The dotted black line shows the area of clivectomy used to create a small bone window. FM = foramen magnum; $\mathrm{OC}=$ occipital condyle. $\mathrm{E}$ : The dotted black line in the dry skull model indicates a small bone window. F: Surgical view of the ventral aspect of the brainstem. The black arrowheads indicate the 4 points of ligation used to induce BAO. ACBs, AICAs, and the lateral mesencephalic veins $\left({ }^{* *}\right)$ and branches $\left({ }^{*}\right)$ are visible. G: Schematic illustration of ligation sites used to induce BAO (black arrowheads). The dotted line shows the surgical area in panel F. Bars $=1 \mathrm{~cm}(\mathrm{~A}-\mathrm{C}$ and $\mathrm{E}) ; 1 \mathrm{~mm}(\mathrm{D}$ and $\mathrm{F})$. 

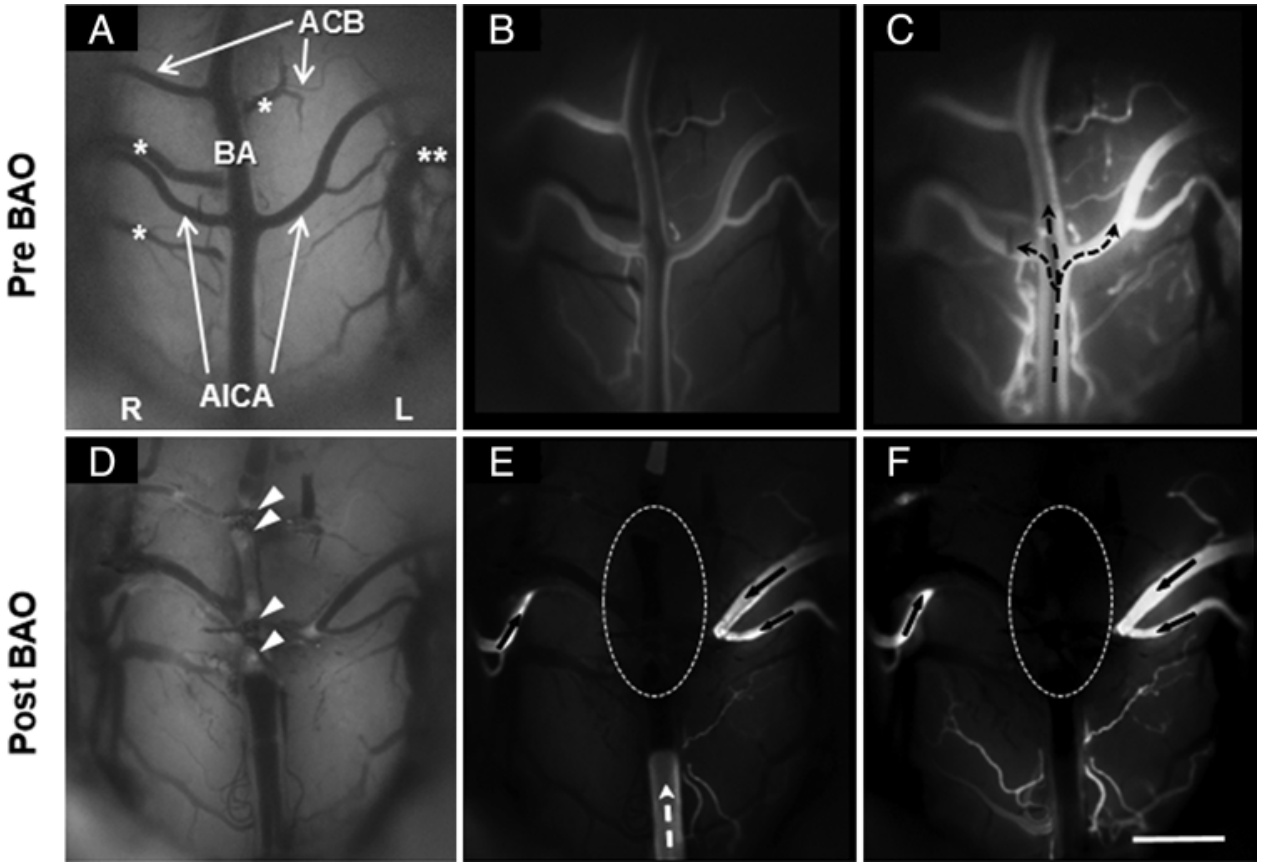

FIG. 2. Flow images obtained before $(A-C)$ and after $(D-F)$ the $B A O$. $A$ : The proximal segments of the $B A$ and $A I C A$, the $A C B s$, and lateral mesencephalic vein $\left({ }^{* *}\right)$ before injection of FITC-dextran. ${ }^{*}$ Branches of the lateral mesencephalic vein. B: Immediately after tail vein injection of FITC-dextran. C: Late arterial phase showing anterograde flow from the proximal BA to the AICA, ACB, and distal BA. D: Sites of the 4 points of the BAO with 10-0 nylon monofilament (white arrowheads). E: Immediately after tail vein injection of FITC-dextran after the BAO. Fluorescent images in the early arterial phase show no-flow areas due to BAO (white oval), indicating the infarct. Retrograde refilling from the circle of Willis and/or AICA (black arrows) is visible. At the bottom of the occlusion, a back-and-forth movement of a pool of blood within the vessel was observed with vascular brain pulsation, but it did not flow to the occluded BA (white arrow). F: Late arterial phase showing the no-flow area (white oval). Black arrows show retrograde refilling. $B a r=1 \mathrm{~mm}$.

a back-and-forth movement of a pool of blood within the vessel was observed with vascular brain pulsation, but it did not flow to the occluded BA (Fig. 2E). Retrograde refilling from the circle of Willis and/or AICA was observed (Fig. 2E and F). To prevent postoperative spinal fluid leakage after BAO, closure of the bone window was accomplished using a small amount of autologous subcutaneous fat. The overall survival rate was $71 \%$ (30/42).

Thirty BAO rats were used in this study. Seven rats were in the MSC infusion group, and another 7 rats were in the vehicle group. The survival time for these animals, during which behavioral, MRI, and histological examination with $\mathrm{H} \& \mathrm{E}$ staining was performed, was 28 days. The remaining 16 rats were used for the immunohistochemical analysis. The BAO rats with GFP-MSC infusions survived for 3 days, 14 days, and 28 days (4 rats/time point) and rats with wild type-derived MSC infusions survived for 3 days (4 rats).

\section{Preparation of MSCs From Rat Bone Marrow}

The methodology of preparing an MSC culture was based on our previous studies. ${ }^{20,21,22,29}$ In brief, bone marrow was obtained from the femoral bones of adult rats or GFP-expressing rats (W-Tg[CAG-GFP]184Ys). The marrow was then diluted to $20 \mathrm{ml}$ with DMEM (Sigma-Aldrich) and supplemented with $10 \%$ heat-inactivated fetal bovine serum (Thermo Fisher Scientific Inc.), $2 \mathrm{mM} \mathrm{L-}$ glutamine (Sigma-Aldrich), $100 \mathrm{U} / \mathrm{ml}$ penicillin, and 0.1 $\mathrm{mg} / \mathrm{ml}$ streptomycin (Thermo Fisher Scientific Inc.) and incubated for 3 days $\left(5 \% \mathrm{CO}_{2}, 37^{\circ} \mathrm{C}\right)$. When the cultures almost reached confluence, the adherent cells were detached using a trypsin-EDTA solution (Sigma-Aldrich) and subcultured in a $150-\mathrm{mm}^{2}$ tissue culture dish (surface area: $148 \mathrm{~cm}^{2}$, Iwaki) with $5 \times 10^{5}$ cells/ml and $15 \mathrm{ml}$ of culture medium; thus, the plating density was approximately $3.5 \times 10^{3} / \mathrm{cm}^{2}$. MSCs were used for in vivo injection at 3 passages.

\section{MSC Infusion Procedures}

Transplantation protocols consisted of 2 groups. The initial T2-weighted MR images were obtained, and ischemic lesions in the brainstem were confirmed 1 day after the induction of BAO. The BAO animals were divided randomly into the MSC-treated group $(\mathrm{n}=7)$ and vehicle group $(n=7)$. The rats were intravenously infused with the bone marrow-derived MSCs $\left(1.0 \times 10^{6}\right.$ cells in $1 \mathrm{ml}$ of fresh DMEM) or medium alone in the vehicle group (1 ml of fresh DMEM) via the left femoral vein. We confirmed the high cell viability (> 99\%) with $0.4 \%$ trypan blue immediately after the procedure. All rats, including those in the vehicle group, were injected daily with cyclosporine A $\left(10 \mathrm{mg} / \mathrm{kg}\right.$ intraperitoneally). ${ }^{22,29,34}$

\section{MRI Studies and Measurement of Infarct Volume}

MRI was performed as previously described..$^{22,29,34}$ 
The rats were anesthetized with ketamine $(75 \mathrm{mg} / \mathrm{kg}$ ) and xylazine $(10 \mathrm{mg} / \mathrm{kg}$ intraperitoneally). Each rat was then placed in an animal holder/MRI probe apparatus and positioned inside the magnet with its head held in place inside the imaging coil. All MRI measurements were performed using a 7-T, 18-cm-bore superconducting magnet (Oxford Magnet Technologies) interfaced with a UNITY INOVA console (Oxford Instruments and Varian Inc.).

In brief, T2-weighted images were obtained from a $1.0-\mathrm{mm}$-thick coronal section with a $0.5-\mathrm{mm}$ gap using a $30 \times 30-\mathrm{mm}$ field of view (TR $3000 \mathrm{msec}$, TE $30 \mathrm{msec}$ ) and reconstructed using a $256 \times 256$ image matrix. Accurate brain positioning was ensured to center all slices 5 $\mathrm{mm}$ posterior to the rhinal fissure with the head of the rat held in a flat skull position. The images were obtained 1 and 28 days after BAO (7 rats/group).

The ischemic lesion area was calculated from each T2-weighted image using imaging software (Scion Image v4.0.2 beta, Scion Corp.) based on the method described in previous reports. ${ }^{23,29,34}$ Briefly, after optimal adjustment of the contrast, the edges of the lesions were manually traced on each of the 1 or 2 coronal slices that completely covered the occluded BA territory in each animal. The signal intensity of the lesion was 1.25 times higher than that of the remaining intact area around the lesion in the brainstem. To calculate the lesion volume, the hyperintense areas were then summed and multiplied by the slice thickness plus the interslice gap.

\section{Histological Analysis}

At 28 days after occlusion, the BAO rats $(n=7 /$ group $)$ were deeply anesthetized with an overdose of ketamine $(100 \mathrm{mg} / \mathrm{kg})$ and xylazine $(20 \mathrm{mg} / \mathrm{kg}$ intraperitoneally) and then perfused with $4 \%$ paraformaldehyde after phosphate-buffered saline. The rat brains were removed and processed for standard frozen sectioning. Coronal cryosections were sliced into $30-\mu \mathrm{m}$-thick sections using a cryostat at the bregma approximately $-9.5 \mathrm{~mm}$ to -13.0 $\mathrm{mm}$ through the infarcted area with no intervals. Routine $\mathrm{H} \& \mathrm{E}$ staining was used to delineate the extent of the ischemic lesion for detection of a pale pink region. Images were acquired using a microscope (BZ-X710, Keyence Corp.) with a $4 \times$ objective. To calculate the volume of the infarct area $\left(\mathrm{mm}^{3}\right)$ in an entire brainstem section, we used the BZ-X Analyzer software's merge function (Keyence Corp.), and the software was used to manually delineate and automatically measure the area of the pale pink region. The infarct area $\left(\mathrm{mm}^{2}\right)$ of each slice was summed to calculate the total infarct volume $\left(\mathrm{mm}^{3}\right)$. For the immunohistochemical analysis and to detect GFP-MSCs, the rats were euthanized 3 days $(n=4), 14$ days $(n=4)$, and 28 days $(n=4)$ after the MSC injection as previously described. ${ }^{20,22,29}$ To determine whether autofluorescence of the MSCs was present at the wavelengths used to study GFP fluorescence, we also examined these sections of the $\mathrm{BAO}$ rats infused with MSCs derived from wild-type rats killed 3 days after infusion $(n=4)$. The 20 - $\mu$ m-thick cryosections were processed to detect GFP (dilution 1:1000, ab13970, Abcam). After being washed in PBS-Tween 20 solution 4 times, the sections were incubated in secondary antibody AF 488-conjugated goat anti-chicken immuno- globulin Y (1:1000; ab150173, Abcam). To analyze the cell fate of infused GFP-MSCs, double-labeling studies were performed with the use of antibodies to neurons (monoclonal mouse anti-NeuN, clone A60 antibody, 1:400; MAB377, EMD Millipore) and astrocytes (monoclonal rabbit anti-GFAP, 1:600; ab33922, Abcam). After being washed in PBS-Tween 20 solution 4 times, the sections were incubated in secondary antibody AF 488-conjugated goat anti-chicken immunoglobulin Y for GFP (1:1000; ab150173 Abcam), goat anti-mouse IgG Alexa Fluor 594 for NeuN (1:500; A-11032, Invitrogen), and goat anti-rabbit IgG, Alexa Fluor 594 for GFAP (1:500; A-11037, Invitrogen); counterstained with DAPI; and coverslipped with VECTASHIELD (Vector Laboratories). The sections were examined using confocal microscopy with Ex/Em (405 nm for DAPI, $488 \mathrm{~nm}$ for GFP, and $561 \mathrm{~nm}$ for NeuN or GFAP; LSM780 ELYRA S.1 system, Zeiss).

\section{Treadmill Stress Test}

The treadmill stress test was performed as described previously. ${ }^{11,22,24,29,38}$ Before $\mathrm{BAO}$, the rats (7 rats/group) were trained to run on a motor-driven treadmill (Muromachi Inc.) $20 \mathrm{~min} /$ day for 2 days/wk at a speed of $20 \mathrm{~m} / \mathrm{min}$ with a slope of $20^{\circ}$. The rats were placed on the moving belt facing away from an electrified grid and induced to run in the direction opposite the belt's movement. Thus, to avoid foot shocks (1.0-mA intensity), the rats had to move forward. Only the rats that had learned to avoid the mild electrical shock were included in this study. The maximum speed at which the rats could run on a motor-driven treadmill was recorded on day 1 before MSC injection and on days $7,14,21$, and 28 after BAO.

\section{Modified Neurological Severity Scores}

The modified neurological severity scores (mNSSs) were determined as previously described ${ }^{6,30}$ The rat's neurological function was graded on a scale of 0-18 (normal score, 0 ; maximum deficit score, 18) 1 day before the MSC injection and on days 7, 14, 21, and 28 after BAO (7 rats/ group). The mNSS is a composite of motor (muscle status and abnormal movement), sensory (visual, tactile, and proprioceptive), reflex, and balance tests. The reflexes tested in the mNSS are the pinna and startle reflexes, which may be affected by brainstem damage. In the mNSS, a score of 1 is awarded for an inability to perform the test or lack of a tested reflex and an overall composite score is given to determine impairment; thus, the higher the score, the more severe the injury.

\section{Beam Walk Test}

The beam walk test was performed as previously described..$^{5,17,32,33}$ A 130 -cm-long wooden beam with a 15 -mm-wide flat surface was elevated $40 \mathrm{~cm}$ above the floor. The ability to balance was scored, with steady posture as a score of 0 ; grasping the side of the beam as 1 ; hugging the beam and 1 limb falling down from it as 2; hugging the beam and 2 limbs falling down from the beam, spinning on the beam, or taking longer than $60 \mathrm{sec}-$ onds to cross as 3; attempting to balance on the beam but falling off within 40 seconds as 4 ; and falling off within 

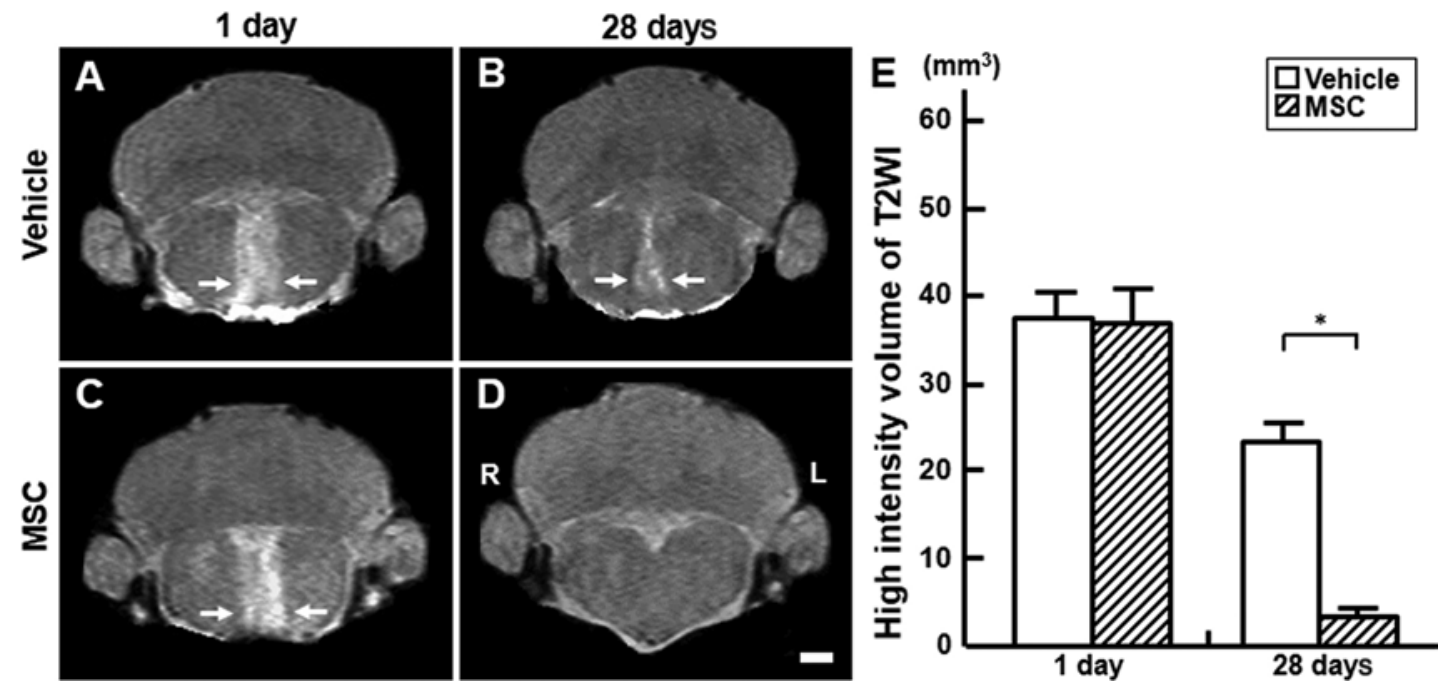

FIG. 3. Evaluation of the ischemic lesion volume on T2-weighted MR images (T2WI). The images were obtained on day 1 (A) and day 28 (B) after BAO in the vehicle group and day 1 (C) and day 28 (D) after BAO in the MSC group. Bar = $5 \mathrm{~mm}$. E: Summary of lesion volumes seen on the images. Arrows in panels A-C indicate the edges of the infarcted lesion. Bars indicate the mean and error bars the SEM. * $p<0.0001$.

20 seconds as 5 . Rats that were unable to maintain balance, immediately fell off the beam, or hung onto the beam received a score of 6 ( 7 rats/group).

\section{Statistical Analysis}

All statistical analyses were performed using JMP Pro 12.2.0 (SAS Institute Inc.). Comparison of the experimental and control groups was performed using the Student t-test. Significance levels were designated $p<0.01$ (behavioral function) or $\mathrm{p}<0.001$ (histology). Mean values are presented as the mean \pm SEM.

\section{Results}

\section{Ischemic Lesion Volume by MRI Analysis}

The ischemic lesion volume was estimated for the MSC and vehicle groups using in vivo MRI (see MRI Studies and Measurement of Infarct Volume). T2-weighted images were obtained on days 1 and 28 after BAO (Fig. 3). The images obtained on day 1 before MSC injection or vehicle confirmed no intergroup difference in the initial stroke volume (MSC group, $35.75 \pm 12.53 \mathrm{~mm}^{3}$ and vehicle group, $36.37 \pm 9.60 \mathrm{~mm}^{3} ; \mathrm{p}=0.89$ ). The lesion volume $\left(\mathrm{mm}^{3}\right)$ was determined by analyzing the T2-weighted images and high-intensity areas on serial images collected through the brainstem. On day 28, the MSC-treated group exhibited a greater reduction in lesion volume than the vehicle group (MSC group, $3.25 \pm 2.39 \mathrm{~mm}^{3}$ and vehicle group, $30.32 \pm 6.33 \mathrm{~mm}^{3} ; \mathrm{p}<0.0001$ [Fig. 3]). Thus, the intravenous infusion of MSCs reduced the ischemic lesion volume.

\section{Ischemic Lesion Volume by Histological Determination}

A second independent measure of ischemic lesion volume was performed using $\mathrm{H} \& \mathrm{E}$ stains, which were used to determine the infarct volume 28 days after BAO. The infarct appeared well demarcated around the midline of the brainstem and allowed volume calculation (Fig. 4A and $\mathrm{B})$. The mean volume for the MSC-treated group $\left(0.92 \pm 0.95 \mathrm{~mm}^{3}\right)$ was on average markedly smaller than that of the vehicle group $\left(16.58 \pm 5.07 \mathrm{~mm}^{3}\right)$ on day $28(\mathrm{p}$ $<0.0001$, Fig. 4C). As with data from the MRI analysis, there was a substantial reduction in infarct volume with the intravenous infusion of MSCs in this BAO model system.

\section{Behavioral Function}

To assess behavioral performance in the experimental groups, a treadmill stress test, mNSS, and beam walk test (included in the mNSS) were performed on day 1 before the MSC injection and on days 7, 14, 21, and 28 after BAO. Prior to $\mathrm{BAO}$, the rats performed similarly in all tests.
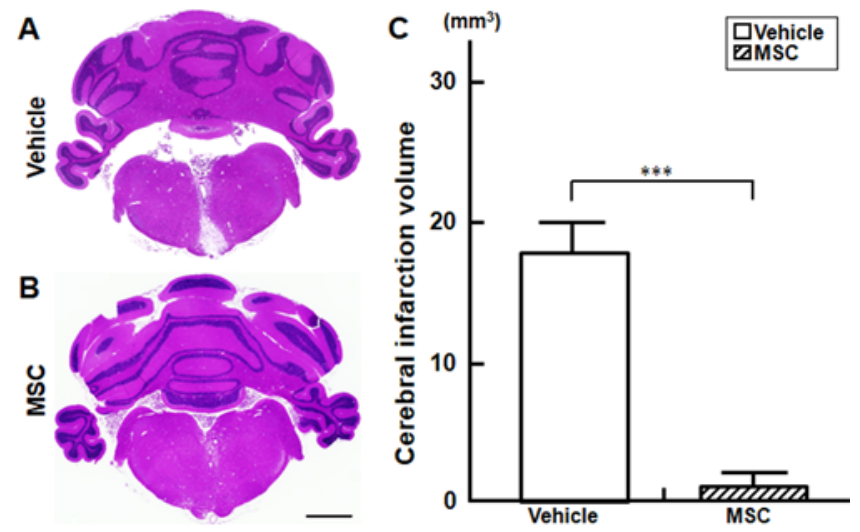

FIG. 4. Light microscopic image of the brainstem and cerebellum in the vehicle group (A) and MSC group (B) 28 days after BAO. H \& E. C: Histogram showing the summary of infarct volume. Bars indicate the mean and error bars the SEM. Bar $=2 \mathrm{~mm}(\mathrm{~A}$ and $\mathrm{B}) .{ }^{* * *} \mathrm{p}<0.0001$. 
In the treadmill stress test, the maximum speed at which the rats could run on a motor-driven treadmill was recorded. One day after $\mathrm{BAO}$, the maximum velocity on the treadmill test was at its maximum deficit, and there was no significant difference between the 2 experimental groups (7 rats/group). Although both groups of rats showed gradually improved functional recovery up to 28 days, MSC-treated rats showed a greater functional recovery than rats in the vehicle group. These results are summarized in Fig. 5.

Assessment of the mNSS indicated no significant improvement in scores at 1 day after BAO in the MSC-treated and vehicle groups; however, in the MSC-treated group, the improvements were statistically greater over time (7, 14,21 , and 28 days) compared with those of the vehicle group ( $\mathrm{p}<0.05$, Fig. $6 \mathrm{~A})$.

The beam balance test was used to test distinct neurological deficits in posterior circulation stroke to retain posture and balance over the beam. The beam balance test scores were severely impaired 1 day after BAO in both groups, but they significantly improved gradually $(7,14$, 21 , and 28 days) in the MSC-treated group. The ability to balance or retain posture may be the main factor to evaluate in the BAO model (Fig. 6B).

\section{Fate of Infused GFP-MSCs In Vivo}

Histological analysis at 3,14, and 28 days after GFPMSC (green) infusion indicated survival of the infused GFP-MSCs and distribution to the infarct (Fig. 7A). There were few GFP-MSCs at the ischemic lesion boundary. There were no green cells observed in the sections of rats infused with MSCs derived from wild-type rats (Fig. 7B). The percentage of intravenously infused GFP-MSCs accumulated in the lesion was approximately $7.4 \%$ (day 3), $1.7 \%$ (day 14), and $0.5 \%$ (day 28). Moreover, immunohistochemical analysis revealed that the percentage of GFAPpositive GFP-MSCs was $0 \%$ (day 3), $1.3 \%$ (day 14), and $0.28 \%$ (day 28), and that of NeuN-positive GFP-MSCs was $0 \%$ (day 3 ), $0.3 \%$ (day 14 ), and $0.28 \%$ (day 28 ).

\section{Discussion}

In the present study, we established a persistent BAO model confirmed by both MRI and $\mathrm{H}$ \& E staining and then showed that the intravenous administration of MSCs into this BAO model improved behavioral function and reduced the infarct volume.

Wojak et al..$^{39}$ coagulated the BA through the transclival window at 2 points caudal and rostral to or straddling the AICA and concluded that the 2-point BAO can produce brainstem infarction; however, the infarct size and nature depend on the rostrocaudal occlusion position. ${ }^{39}$ The BAO model in this study was induced by selective ligation of 4 points of the proximal BA (just distal and proximal to the origin of the AICA) and anterior cerebellar arteries approximately $1 \mathrm{~mm}$ apart from the AICA through the transclival window using 10-0 nylon monofilament suture. We confirmed no anterograde or retrograde blood flow from the BA and retrograde filling from the collateral circulation via the ACBs and AICA at 5-10 minutes after the occlusion by injecting FITC-dextran and using a fluores-

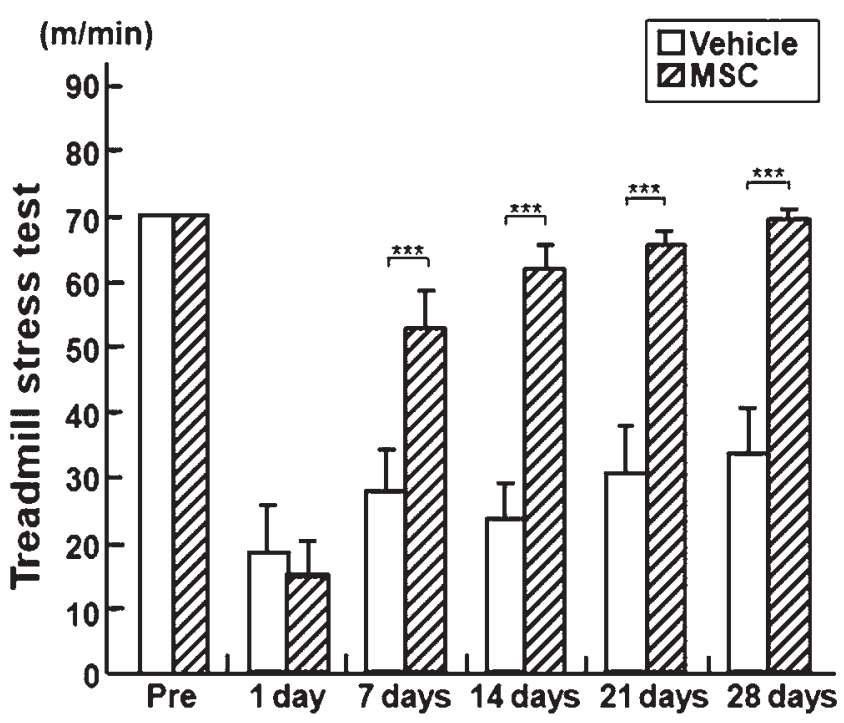

FIG. 5. Treadmill stress test. The maximum speed at which the rats could run on a motor-driven treadmill was recorded. There were no statistically significant intergroup differences 1 day after BAO; however, the MSC group showed greater functional improvement than the vehicle group on days $7,14,21$, and 28 . Bars indicate the mean and error bars the SEM. ${ }^{* * *} p<0.0001$.

cence microscope. The absence of retrograde filling to the occluded BA suggests that the proximal part of the BA was completely occluded and there was little influence from the collateral flow from the carotid system through the posterior communicating artery. The persistent infarcted area was confirmed with MRI and $\mathrm{H} \& \mathrm{E}$ staining. A pale stained area in the $\mathrm{H} \& \mathrm{E}$ sections is indicative of a lesion caused by infarction and is approximately identical in location to the high-intensity brainstem region on T2weighted MRI. The vascular supply of the infarcted area was from the proximal part of the BA. Ischemic volumes calculated by MRI and $\mathrm{H} \& \mathrm{E}$-stained sections were consistent among the animals. The overall survival rate was $71 \%$ (30/42). Thus, this newly developed BAO model system is unique and reproducible for studying brainstem ischemia in rats.

The human brainstem has a higher proportion of white matter than gray matter. ${ }^{7}$ Although rat MCAO models, where the main ischemic region is gray matter, have been widely used for stroke studies with MSCs, ${ }^{2}$ there are few reliable animal models of white matter ischemia. In this study, we established a persistent BAO model as a posterior infarction stroke model in which abundant white matter tracts are located, and showed the therapeutic efficacy of the intravenous infusion of MSCs.

The therapeutic efficacy of the intravenous administration of MSCs was examined in this model system. MRI and $\mathrm{H} \& \mathrm{E}$ staining confirmed a reduced ischemic lesion volume in the MSC group compared with the vehicle group 28 days after MSC infusion. In the $\mathrm{H} \& \mathrm{E}$-stained sections 28 days after infusion, areas of pale staining were still observed in the central parts of the pons through the medulla in the vehicle group, but the same was not observed in the MSC-treated group. These observations 

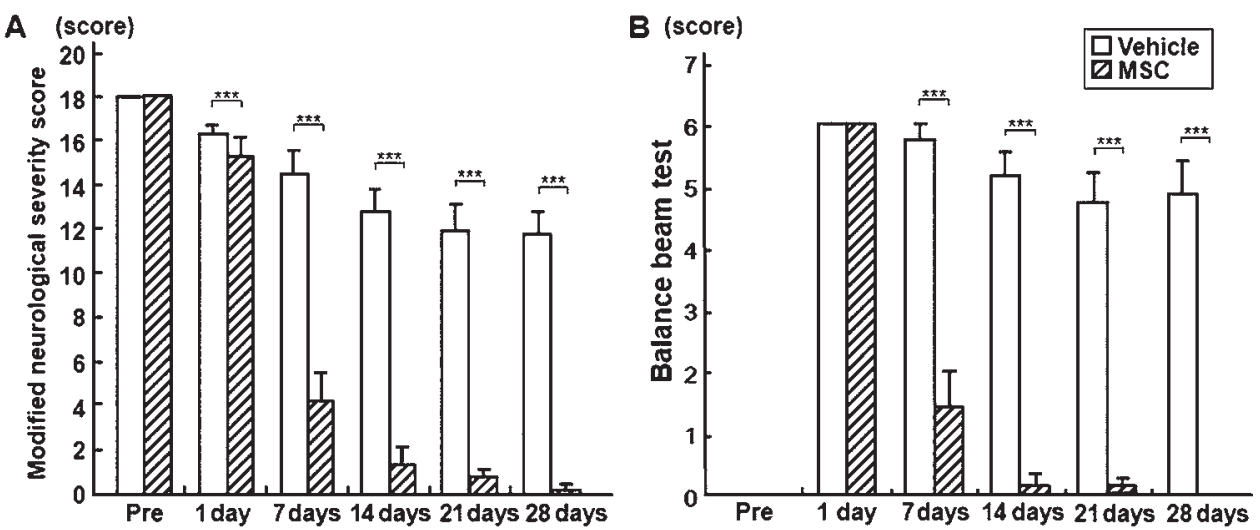

FIG. 6. Modified NSSs and beam walk test scores. For both test scores, there were no statistically significant intergroup differences 1 day after BAO; however, the MSC group showed greater functional improvement than the vehicle group on days 7, 14, 21, and 28. A: The mNSS is a composite of motor (muscle status and abnormal movement), sensory (visual, tactile, and proprioceptive), reflex, and balance tests. In the severity scores of functional deficits, the rat's neurological function was graded on a scale of $0-18$. The higher the score, the more severe the functional deficits. B: The beam walking test rates fine motor coordination and balance, which was graded on a score of $0-6$. Rats with no disability were given a score of 0 , while those that immediately fell off were given a score of 6 . Bars indicate the mean and error bars the SEM. ${ }^{* * *} p<0.0001$.

showed that ischemic injury to the brainstem was reduced by MSC infusion.

Functional outcomes were examined using 3 behavioral tests over a period of 28 days after the MSC infusion. The treadmill stress test was used to evaluate locomotion activity. The mNSS was to test motor activity composition (muscle status and abnormal movement), sensory (visual, tactile, and proprioceptive), reflexes, and balance. Although a beam walking assessment is included in the mNSS, we analyzed this score independently to detect the deficit in fine motor coordination and balance due to the brainstem infarction. With these multiple behavioral tests, including the beam walk, the animals that received an intravenous infusion of MSCs displayed greater behavioral ability than the vehicle group, suggesting that infused MSCs provide therapeutic efficacy in brainstem ischemia. These results indicate that infused MSCs have therapeutic effects not only on the cerebral ischemia tested by the MCAO model ${ }^{9-11,21,22,29,34}$ but also on brainstem ischemia. Within the brainstem region of ischemia in this model, several important descending motor tracts, including corticospinal, corticobulbar, and rubrospinal, are represented. Important ascending pathways, including the medial lemniscus and lateral spinal thalamic tracts, are also within the lesion area. Ischemic damage to these important descending and ascending pathways likely accounts for the motor and sensory deficits.

Previous studies have shown an accumulation of intravenously administered MSCs in the injured region in diseases of the CNS, including stroke, ${ }^{16,20,22,29}$ associated with beneficial effects. It has been suggested that the MSCs have a tendency to home to the damaged lesion sites, where they enhance their therapeutic effectiveness. ${ }^{25}$ The acute accumulation of the intravenously administered GFP-MSCs in this model system and the associated neuroprotection is consistent with those in previous studies. In this study, we focused on the therapeutic efficacy of infused MSCs using a newly developed BAO model. This model is useful to elucidate the precise mechanisms of the beneficial effects of MSCs reported here, which are not fully established.
It should be noted that the potential beneficial effect of MSCs in various models of CNS injury includes induction of axonal sprouting and neovascularization, restoration of the blood-brain barrier, remyelination, and facilitation of synaptogenesis., ${ }^{9} 12,18$

In the clinic, approximately $20 \%$ of all ischemic events in the brain involve the posterior circulation area, ${ }^{3}$ and a posterior circulation stroke is a major determinant of poor prognosis in poststroke patients. ${ }^{19,31}$ Even after advanced treatment with intravenous recombinant tissue plasminogen activator and/or endovascular stroke therapy, the death and disability rates associated with $\mathrm{BAO}$ remain high. ${ }^{1,15,31,37}$

The intravenous infusion of MSCs may represent a novel treatment for patients with infratentorial stroke who were not administered thrombolysis with recombinant tissue plasminogen activator, in whom endovascular recanalization of the occluded artery was not performed, or who arrived out of the therapeutic time window and developed neurobehavioral deficits in the chronic phase of infratentorial ischemia.
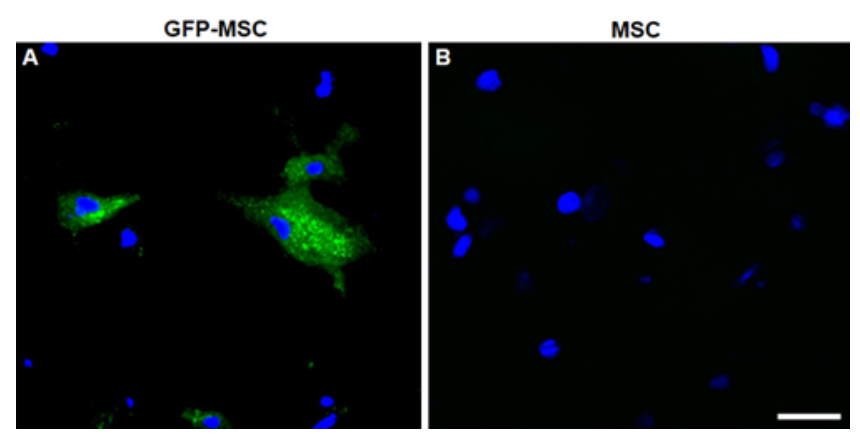

FIG. 7. Confocal microscopy images of the brainstem lesion 3 days after BAO. A: Demonstration of numerous distributed GFP-labeled MSCs (green). Endogenous cells were stained with DAPI (blue). B: On the infusion of non-GFP-MSCs from wild-type rats, no GFP-positive cells were observed. Bar $=250 \mu \mathrm{m}$. 


\section{Conclusions}

In this study, we established a stable BAO model by selectively ligating 4 proximal points of the BA. Intravenous MSC administration reduced the ischemic lesions in the brainstem and improved functional outcomes after BAO in rats.

\section{Acknowledgments}

This work was supported in part by JSPS KAKENHI (grant nos. 17K10901, 17K01513, 16K10730, 15K10365); the AMED Translational Research Network Program (B51); the RR\&D Service of Department of Veterans Affairs (B7335R, B9260L); NMSS (RG2135); and the CT Stem Cell Research Program (12-SCBYale-05). We are thankful to the National BioResource ProjectRat (http://www.anim.med.kyoto-u.ac.jp/NBR/) for providing this strain of rat (NBRP rat no.: 0273, W-Tg[CAG-GFP]184Ys). We thank Drs. Mami Yamaguchi and Michitoshi Kimura for their excellent technical assistance.

\section{References}

1. Arnold M, Nedeltchev K, Schroth G, Baumgartner RW, Remonda L, Loher TJ, et al: Clinical and radiological predictors of recanalisation and outcome of 40 patients with acute basilar artery occlusion treated with intra-arterial thrombolysis. J Neurol Neurosurg Psychiatry 75:857-862, 2004

2. Atchaneeyasakul K, Watanabe M, Zhang T, Lord S, Guada L, Bhattacharya P, et al: Effects of intra-arterial delivery of mesenchymal stem cells on infarct size and neurological outcome in rat middle cerebral artery occlusion model: a systematic review and meta-analysis. Neurology 88 (16 Suppl):P4.216, 2017 (Abstract)

3. Bamford J, Sandercock P, Dennis M, Burn J, Warlow C: Classification and natural history of clinically identifiable subtypes of cerebral infarction. Lancet 337:1521-1526, 1991

4. Bristow MS, Simon JE, Brown RA, Eliasziw M, Hill MD, Coutts SB, et al: MR perfusion and diffusion in acute ischemic stroke: human gray and white matter have different thresholds for infarction. J Cereb Blood Flow Metab 25:1280-1287, 2005

5. Carter RJ, Morton J, Dunnett SB: Motor coordination and balance in rodents. Curr Protoc Neurosci Chapter 8:Unit $8.12,2001$

6. Chen J, Li Y, Wang L, Zhang Z, Lu D, Lu M, et al: Therapeutic benefit of intravenous administration of bone marrow stromal cells after cerebral ischemia in rats. Stroke 32:10051011, 2001

7. Fisher M, Feuerstein G, Howells DW, Hurn PD, Kent TA, Savitz SI, et al: Update of the stroke therapy academic industry roundtable preclinical recommendations. Stroke 40:2244-2250, 2009

8. Fluri F, Schuhmann MK, Kleinschnitz C: Animal models of ischemic stroke and their application in clinical research. Drug Des Devel Ther 9:3445-3454, 2015

9. Honmou O, Houkin K, Matsunaga T, Niitsu Y, Ishiai S, Onodera R, et al: Intravenous administration of auto serum-expanded autologous mesenchymal stem cells in stroke. Brain 134:1790-1807, 2011

10. Honmou O, Onodera R, Sasaki M, Waxman SG, Kocsis JD: Mesenchymal stem cells: therapeutic outlook for stroke. Trends Mol Med 18:292-297, 2012

11. Iihoshi S, Honmou O, Houkin K, Hashi K, Kocsis JD: A therapeutic window for intravenous administration of autologous bone marrow after cerebral ischemia in adult rats. Brain Res 1007:1-9, 2004

12. Kocsis JD, Honmou O: Bone marrow stem cells in experimental stroke. Prog Brain Res 201:79-98, 2012
13. Lekic T, Ani C: Posterior circulation stroke: animal models and mechanism of disease. J Biomed Biotechnol 2012:587590, 2012

14. Lekic T, Zhang JH: Posterior circulation stroke and animal models. Front Biosci 13:1827-1844, 2008

15. Lindsberg PJ, Mattle HP: Therapy of basilar artery occlusion: a systematic analysis comparing intra-arterial and intravenous thrombolysis. Stroke 37:922-928, 2006

16. Liu H, Honmou O, Harada K, Nakamura K, Houkin K, Hamada H, et al: Neuroprotection by PIGF gene-modified human mesenchymal stem cells after cerebral ischaemia. Brain 129:2734-2745, 2006

17. Luong TN, Carlisle HJ, Southwell A, Patterson PH: Assessment of motor balance and coordination in mice using the balance beam. J Vis Exp (49):2376, 2011

18. Matsushita T, Lankford KL, Arroyo EJ, Sasaki M, Neyazi M, Radtke C, et al: Diffuse and persistent blood-spinal cord barrier disruption after contusive spinal cord injury rapidly recovers following intravenous infusion of bone marrow mesenchymal stem cells. Exp Neurol 267:152-164, 2015

19. Mattle HP, Arnold M, Lindsberg PJ, Schonewille WJ, Schroth G: Basilar artery occlusion. Lancet Neurol 10:1002-1014, 2011

20. Morita T, Sasaki M, Kataoka-Sasaki Y, Nakazaki M, Nagahama H, Oka S, et al: Intravenous infusion of mesenchymal stem cells promotes functional recovery in a model of chronic spinal cord injury. Neuroscience 335:221-231, 2016

21. Nakamura H, Sasaki Y, Sasaki M, Kataoka-Sasaki Y, Oka S, Nakazaki M, et al: Elevated brain derived neurotrophic factor (BDNF) levels in plasma but not serum reflect in vivo functional viability of infused mesenchymal stem cells after middle cerebral artery occlusion in rat. J Neurosurg Sci [epub ahead of print], 2017

22. Nakazaki M, Sasaki M, Kataoka-Sasaki Y, Oka S, Namioka $\mathrm{T}$, Namioka A, et al: Intravenous infusion of mesenchymal stem cells inhibits intracranial hemorrhage after recombinant tissue plasminogen activator therapy for transient middle cerebral artery occlusion in rats. J Neurosurg 127:917-926, 2017

23. Neumann-Haefelin T, Kastrup A, de Crespigny A, Yenari MA, Ringer T, Sun GH, et al: Serial MRI after transient focal cerebral ischemia in rats: dynamics of tissue injury, blood-brain barrier damage, and edema formation. Stroke 31:1965-1973, 2000

24. Onda T, Honmou O, Harada K, Houkin K, Hamada H, Kocsis JD: Therapeutic benefits by human mesenchymal stem cells (hMSCs) and Ang-1 gene-modified hMSCs after cerebral ischemia. J Cereb Blood Flow Metab 28:329-340, 2008

25. Ren G, Chen X, Dong F, Li W, Ren X, Zhang Y, et al: Concise review: mesenchymal stem cells and translational medicine: emerging issues. Stem Cells Transl Med 1:51-58, 2012

26. Sasaki M, Honmou O, Kocsis JD: A rat middle cerebral artery occlusion model and intravenous cellular delivery. Methods Mol Biol 549:187-195, 2009

27. Sasaki M, Honmou O, Radtke C, Kocsis JD: Development of a middle cerebral artery occlusion model in the nonhuman primate and a safety study of i.v. infusion of human mesenchymal stem cells. PLoS One 6:e26577, 2011

28. Sasaki M, Radtke C, Tan AM, Zhao P, Hamada H, Houkin K, et al: BDNF-hypersecreting human mesenchymal stem cells promote functional recovery, axonal sprouting, and protection of corticospinal neurons after spinal cord injury. J Neurosci 29:14932-14941, 2009

29. Sasaki Y, Sasaki M, Kataoka-Sasaki Y, Nakazaki M, Nagahama H, Suzuki J, et al: Synergic effects of rehabilitation and intravenous infusion of mesenchymal stem cells after stroke in rats. Phys Ther 96:1791-1798, 2016

30. Schaar KL, Brenneman MM, Savitz SI: Functional assessments in the rodent stroke model. Exp Transl Stroke Med 2:13, 2010 
31. Schonewille WJ, Wijman CAC, Michel P, Rueckert CM, Weimar C, Mattle HP, et al: Treatment and outcomes of acute basilar artery occlusion in the Basilar Artery International Cooperation Study (BASICS): a prospective registry study. Lancet Neurol 8:724-730, 2009

32. Shen LH, Li Y, Chen J, Zhang J, Vanguri P, Borneman J, et al: Intracarotid transplantation of bone marrow stromal cells increases axon-myelin remodeling after stroke. Neuroscience 137:393-399, 2006

33. Southwell AL, Ko J, Patterson PH: Intrabody gene therapy ameliorates motor, cognitive, and neuropathological symptoms in multiple mouse models of Huntington's disease. J Neurosci 29:13589-13602, 2009

34. Suzuki J, Sasaki M, Harada K, Bando M, Kataoka Y, Onodera R, et al: Bilateral cortical hyperactivity detected by fMRI associates with improved motor function following intravenous infusion of mesenchymal stem cells in a rat stroke model. Brain Res 1497:15-22, 2013

35. Turner DA: Modern Neurosurgery: Clinical Translation of Neuroscience Advances. Boca Raton, FL: CRC Press, 2004

36. Vergouwen MD, Algra A, Pfefferkorn T, Weimar C, Rueckert $\mathrm{CM}$, Thijs V, et al: Time is brain(stem) in basilar artery occlusion. Stroke 43:3003-3006, 2012

37. Weimar C, Goertler M, Harms L, Diener HC: Distribution and outcome of symptomatic stenoses and occlusions in patients with acute cerebral ischemia. Arch Neurol 63:12871291, 2006

38. Whiteley WN, Slot KB, Fernandes P, Sandercock P, Wardlaw $\mathrm{J}$ : Risk factors for intracranial hemorrhage in acute ischemic stroke patients treated with recombinant tissue plasminogen activator: a systematic review and meta-analysis of 55 studies. Stroke 43:2904-2909, 2012

39. Wojak JC, DeCrescito V, Young W: Basilar artery occlusion in rats. Stroke 22:247-252, 1991

\section{Disclosures}

The authors report no conflict of interest concerning the materials or methods used in this study or the findings specified in this paper.

\section{Author Contributions}

Conception and design: M Sasaki, Honmou. Acquisition of data: A Namioka, T Namioka, Oka, Nakazaki, Suzuki, Y Sasaki, Nagahama. Analysis and interpretation of data: M Sasaki, A Namioka, Kataoka-Sasaki. Drafting the article: M Sasaki, A Namioka, Kocsis, Honmou. Critically revising the article: M Sasaki,

Kataoka-Sasaki, Kocsis, Honmou. Reviewed submitted version of manuscript: M Sasaki, Kataoka-Sasaki, Oka, Nakazaki, Onodera, Kocsis, Honmou. Approved the final version of the manuscript on behalf of all authors: M Sasaki. Administrative/technical/material support: M Sasaki, Onodera. Study supervision: M Sasaki, Kocsis, Honmou.

\section{Correspondence}

Masanori Sasaki: Research Institute for Frontier Medicine, Sapporo Medical University School of Medicine, Hokkaido, Japan. msasaki@sapmed.ac.jp. 